\title{
Insulin's direct hepatic effect explains the inhibition of glucose production caused by insulin secretion
}

Dale S. Edgerton, ${ }^{1}$ Guillaume Kraft, ${ }^{1}$ Marta Smith, ${ }^{1}$ Ben Farmer, ${ }^{1}$ Phillip E. Williams, ${ }^{2}$ Katie C. Coate, ${ }^{3}$ Richard L. Printz, ${ }^{1}$ Richard M. O'Brien, ${ }^{1}$ and Alan D. Cherrington ${ }^{1}$

'Department of Molecular Physiology and Biophysics, Vanderbilt University, Nashville, Tennessee, USA. ²Division of Surgical Research, Vanderbilt University School of Medicine, Nashville, Tennessee, USA. ${ }^{3}$ Samford University, Department of Nutrition and Dietetics, Birmingham, Alabama, USA.

Insulin can inhibit hepatic glucose production (HCP) by acting directly on the liver as well as indirectly through effects on adipose tissue, pancreas, and brain. While insulin's indirect effects are indisputable, their physiologic role in the suppression of HCP seen in response to increased insulin secretion is not clear. Likewise, the mechanisms by which insulin suppresses lipolysis and pancreatic $\alpha$ cell secretion under physiologic circumstances are also debated. In this study, insulin was infused into the hepatic portal vein to mimic increased insulin secretion, and insulin's indirect liver effects were blocked either individually or collectively. During physiologic hyperinsulinemia, plasma free fatty acid (FFA) and glucagon levels were clamped at basal values and brain insulin action was blocked, but insulin's direct effects on the liver were left intact. Insulin was equally effective at suppressing HCP when its indirect effects were absent as when they were present. In addition, the inhibition of lipolysis, as well as glucagon and insulin secretion, did not require CNS insulin action or decreased plasma FFA. This indicates that the rapid suppression of HGP is attributable to insulin's direct effect on the liver and that its indirect effects are redundant in the context of a physiologic increase in insulin secretion.

Conflict of interest: The authors have declared that no conflict of interest exists.

Submitted: November 28, 2016 Accepted: February 8, 2017 Published: March 23, 2017

Reference information: JCI Insight. 2017;2(6):e91863. https:// doi.org/10.1172/jii.insight.91863.

\section{Introduction}

The liver plays a critical role in maintaining glucose homeostasis. It is therefore not surprising that insulin regulates hepatic glucose production (HGP) through multiple mechanisms. HGP is inhibited by intracellular signals resulting from the direct binding of insulin to its hepatic receptor (1-6), resulting in a rapid reduction in glycogenolysis (2, 7-9). In addition, insulin can indirectly suppress HGP by several means, including the reduction of circulating free fatty acids (FFAs) and glycerol as a result of the inhibition of lipolysis (10-14), the suppression of glucagon secretion $(15,16)$, and by means of a change in neural input to the liver secondary to insulin action in the brain $(17,18)$.

Because of redundancies in the control of HGP by insulin, each of its various effects are, at least to a degree, dispensable $(5,14,19)$. For example, elevating insulin at the liver alone, both in vivo and ex vivo, rapidly inhibits HGP in multiple species $(2,3,5)$. Although at a minimum basal insulin signaling at the liver is necessary $(14,20)$, a rise in arterial insulin alone, without a change in hepatic insulin levels, can suppress HGP (5) through insulin-mediated suppression of lipolysis (13). Likewise, a change in insulin at the liver is not required for modification of hepatic glucose metabolism by glucagon $(15,21)$ or brain insulin action $(17,18,22)$. Thus, a multitude of studies have shown that HGP can be suppressed in response to either an increase in the arterial or hepatic insulin level, even when there is no change in the other.

What remains unclear is the contextual importance of insulin's multiple effects. Hepatic insulin sensitivity is typically measured in human and rodent studies using the gold-standard clamp approach. This involves infusing insulin into a peripheral vein to create hyperinsulinemia while euglycemia is maintained with glucose infusion. It should be noted, however, that the peripheral route of insulin infusion artificially changes the distribution of the hormone in the blood since in vivo insulin is secreted into the hepatic portal vein. In a normal physiologic setting, therefore, the liver is exposed to 2.5- to 3-fold higher insulin concentrations $(5,13,23-26)$ than the brain, fat, or other tissues. In contrast, when insulin is infused into a 
peripheral vein hepatic insulin levels are $\sim 20 \%$ less than arterial levels. This was demonstrated in a recent rat study in which a low peripheral insulin infusion rate (resulting in only a 2-fold rise in arterial insulin) completely eliminated endogenous insulin secretion and reversed the normal insulin gradient between the liver and peripheral tissues (26). It is apparent, therefore, that when insulin is infused into a peripheral vein its direct effects on the liver are underemphasized, while its indirect effects are exaggerated. Thus, the route of insulin delivery can have a major impact on both the overall response of the liver and the mechanisms by which that response is achieved. Indeed, over a range of insulin doses, peripheral insulin infusion was not as effective at suppressing HGP as compared with direct infusion into the portal vein (26).

Some studies have investigated brain insulin's effects on HGP under so-called basal insulin conditions, when insulin was infused into a peripheral vein to maintain arterial insulin levels at baseline values during somatostatin infusion $(17,18)$. In others, insulin was infused peripherally to create 3-fold arterial hyperinsulinemia $(17,18)$. Because of the loss of the normal hepatic/arterial insulin gradient (which occurs even without somatostatin infusion; see ref. 26), the liver is hypoinsulinemic under such basal conditions and euinsulinemic under the so-called hyperinsulinemic condition $(5,26,27)$. Therefore, it is no surprise that insulin did not directly contribute to the suppression of HGP in many gold-standard clamp studies (11, 17, $18,28,29)$. While such studies demonstrate the capability of insulin to act indirectly, they do not provide insight into the importance of those effects in the context of a physiologic increase in insulin secretion.

It is also important to consider how fasting affects glycogenolytic and gluconeogenic rates in the species being studied. Unlike humans and large animals, which protect glycogen stores and continue to rely on glycogenolysis for many hours after feeding (30-32), rodents progress rapidly into the fasted state, soon becoming totally reliant on gluconeogenesis since there is complete depletion of liver glycogen within a few hours (33). Because the direct and indirect mechanisms by which insulin regulates glycogenolysis and gluconeogenesis differ, care should be taken to ensure appropriate interpretation of data generated from rodents that were fasted for more than a few hours.

In vitro studies have shown that insulin can directly suppress insulin and glucagon secretion, as well as lipolysis, through insulin receptors on $\beta$ cells, $\alpha$ cells, and adipocytes, respectively (34-39). The in vivo regulation of these processes is complex, however, and can include CNS effects. The degree to which insulin per se contributes to the neural control of these processes under physiologic circumstances is currently unclear. Likewise, it is not known how brain insulin-mediated regulation of lipolysis and insulin and glucagon secretion impact the acute regulation of HGP in the context of increased portal vein insulin concentrations.

Therefore, the purpose of this study was to assess whether insulin's acute indirect effects on HGP are additive to, redundant to, or synergistic with its direct hepatic effects, in the context of a physiologic increase in portal vein insulin level in a large animal model. The impact of eliminating each of insulin's indirect effects, either alone or in combination, was determined. We focused on insulin's ability to lower FFAs, activate brain insulin signaling, and reduce glucagon secretion since recent studies have concluded that suppression of lipolysis is the major mechanism by which insulin suppresses HGP (11), that increased brain insulin action is required for the rapid suppression of HGP (17), and because a fall in plasma glucagon is potentially a powerful contributor to insulin's ability to inhibit HGP (15).

\section{Results}

Plasma insulin. Prior to the hyperinsulinemic clamp, the ratio of endogenous insulin in hepatic sinusoidal versus arterial plasma was $2.4 \pm 0.1$ (-90 to 0 minutes; Figures $1 \mathrm{~A}, 2 \mathrm{~A}, 3 \mathrm{~A}$, and $4 \mathrm{~A} ; n=30$ ) due to the secretion of insulin into the hepatic portal vein (the portal vein to arterial insulin ratio was $2.7 \pm 0.2$ ). During portal vein insulin infusion during the clamp ( 0 to 240 minutes) both the arterial and hepatic sinusoidal insulin levels increased $\sim 6$-fold in the control and experimental groups (Figures 1A, 2A, 3A, and 4A). The arterial to hepatic insulin ratio was $2.5 \pm 0.1$ during the clamp in each group, and arterial plasma C-peptide levels fell to below the level of detection of the assay (Figures 1C, 2C, 3C, and 4C), indicating that endogenous insulin secretion was close to completely inhibited by exogenous insulin infusion. This occurred in the absence of somatostatin infusion. Thus, we simulated a 6 -fold rise in insulin secretion, maintaining the physiologic insulin gradient that existed between the liver and the rest of the body prior to the clamp.

Fat clamp group versus control. The arterial plasma glucose levels were similar between the INS (control) group and the fat-clamp (INS+FFA) group (see Methods for descriptions of all study groups) and the glucose infusion rate required to maintain euglycemia was modestly lower at the end of the study in the fat-clamp group (Figure 1B). Hepatic sinusoidal glucagon levels were suppressed similarly in both groups (Figure 1C). 
A

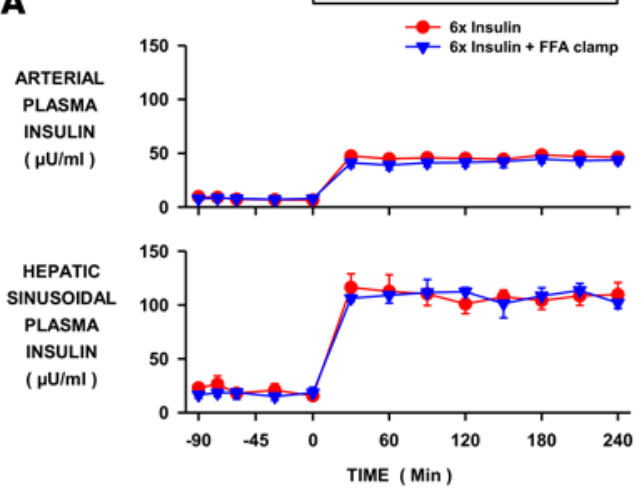

C
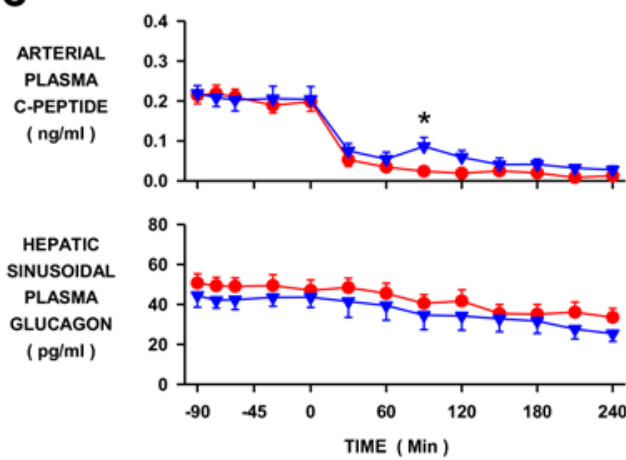

E
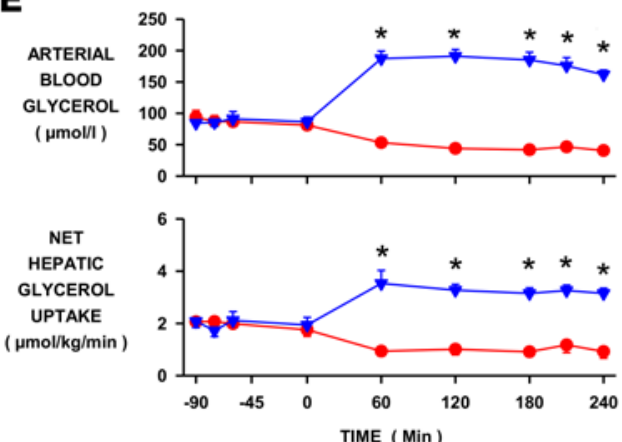

G
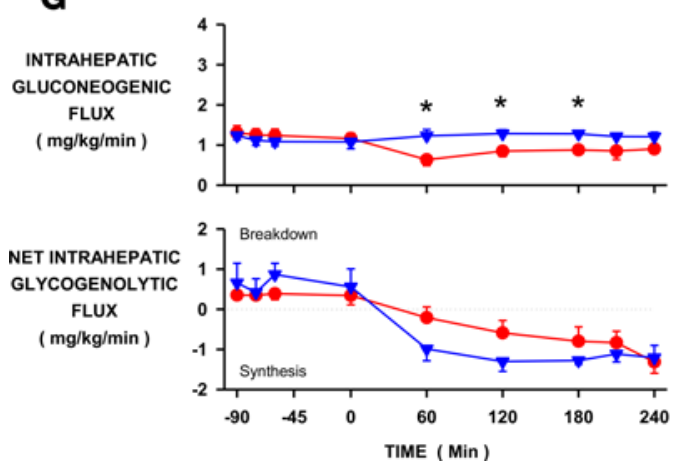

B

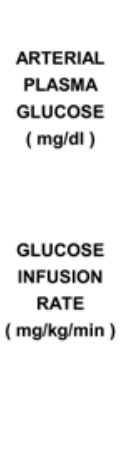

D
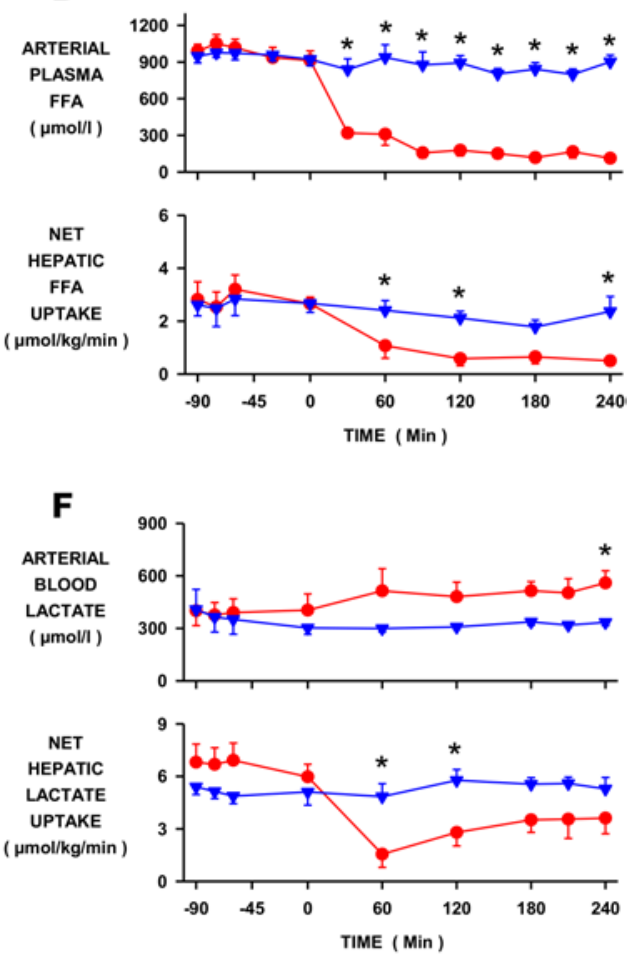

\section{H}
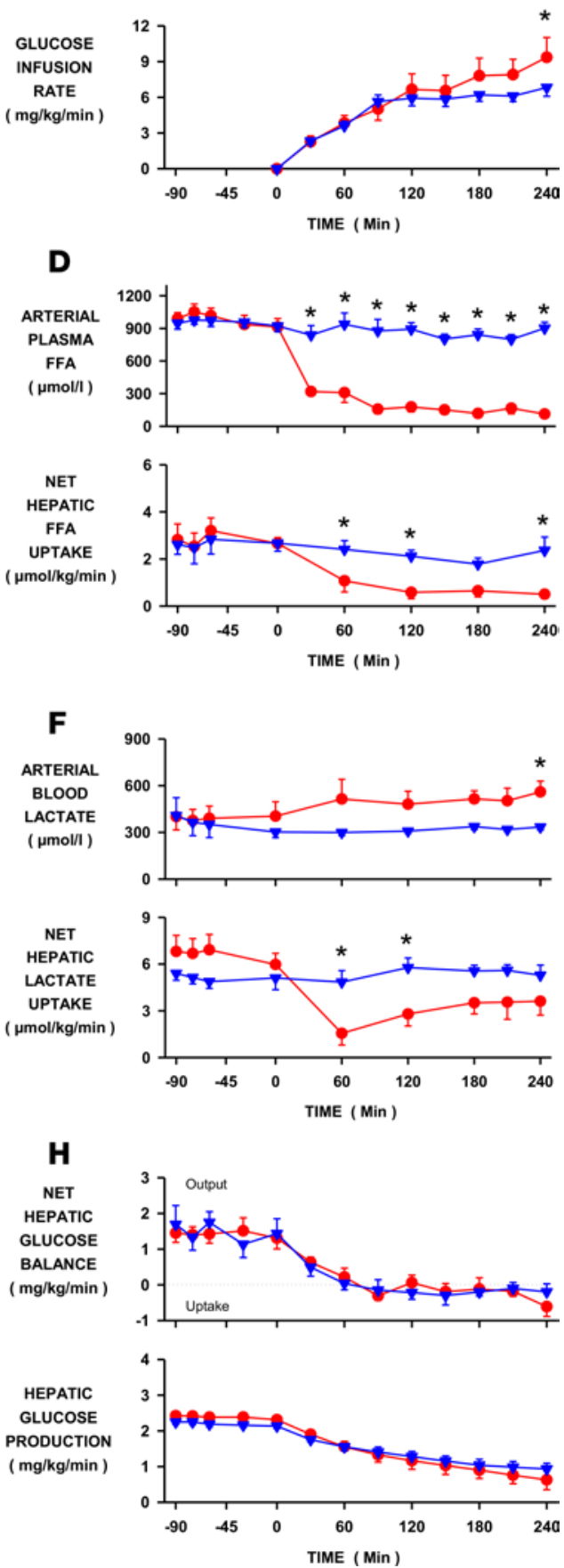

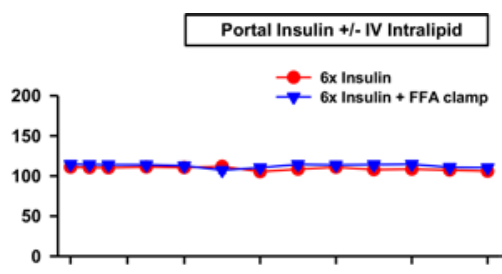

0.05; 2-way repeated measure ANOVA) between groups.

Arterial plasma FFA levels were rapidly reduced by hyperinsulinemia in the INS group, but remained basal in the INS+FFA group when intralipid and heparin were infused (Figure 1D). As a result, net hepatic FFA uptake was either reduced or remained basal, in line with the plasma FFA levels (Figure 1D). Failure to suppress FFA was associated with modestly less stimulation of whole-body glucose utilization (Table 1), which correlated with the reduced need for glucose infusion in the INS+FFA group (Figure 1B). Arterial plasma glycerol levels also declined in the INS group due to insulininduced suppression of lipolysis, but they were elevated 2-fold dur-

ing intralipid infusion (Figure 1E). Liver glycerol uptake is load dependent (unregulated); therefore, the pattern of net hepatic glycerol uptake mirrored plasma glycerol concentrations (Figure 1E). Net hepatic lactate uptake was reduced in the INS group, thereby tending to elevate arterial blood lactate levels, whereas both remained at baseline in the absence of a fall in FFA (Figure 1F). When FFA fell there was a decrease in gluconeogenic flux to glucose-6-phosphate (G6P), which in turn reduced the availability of substrate for glycogen synthesis (Figure $1 G)$. On the other hand, when the FFA levels were prevented from falling, intrahepatic gluconeogenic flux 
A

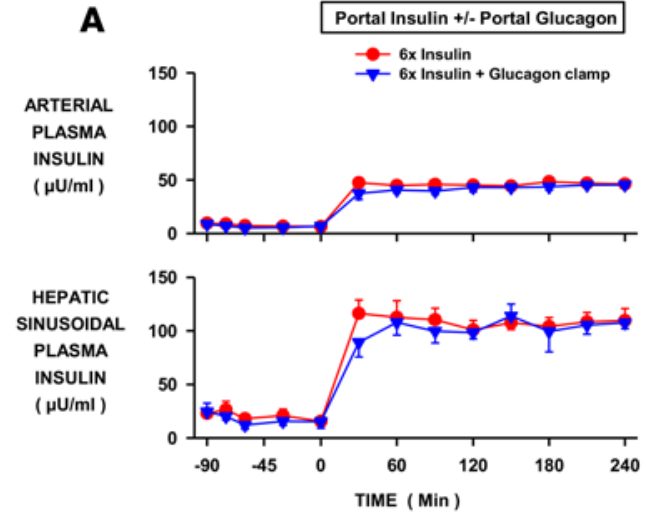

C
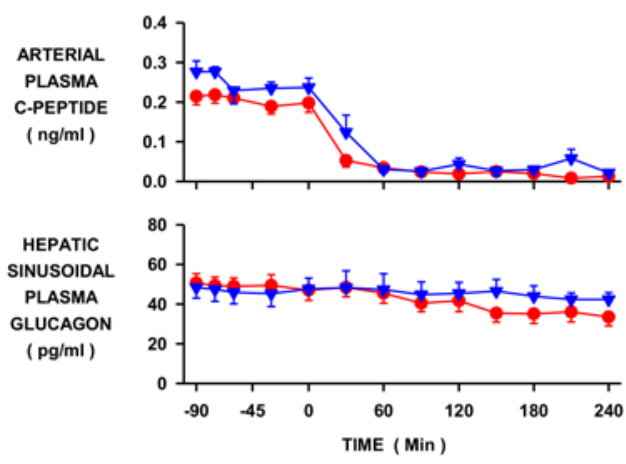

E
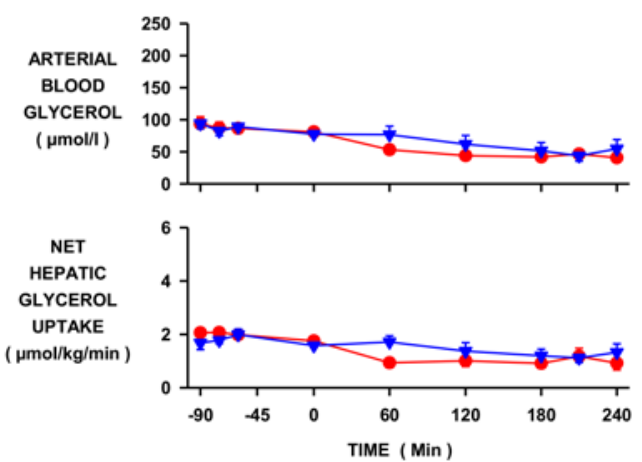

G

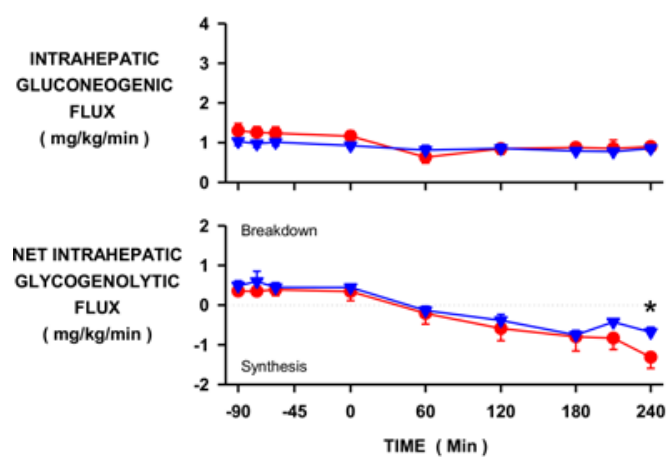

B

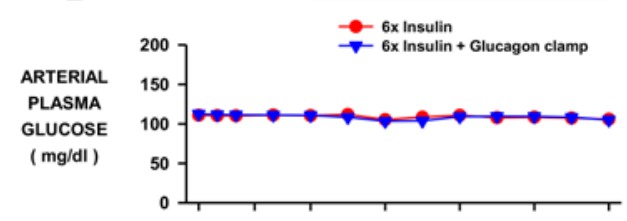
GLUCOSE INFUSION
RATE ( $\mathrm{mg} / \mathrm{kg} / \mathrm{min}$ )

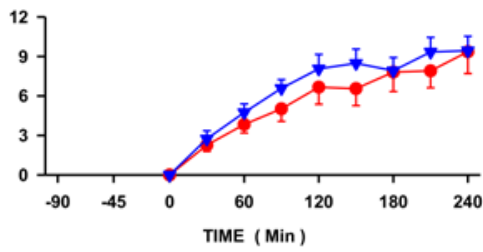

D
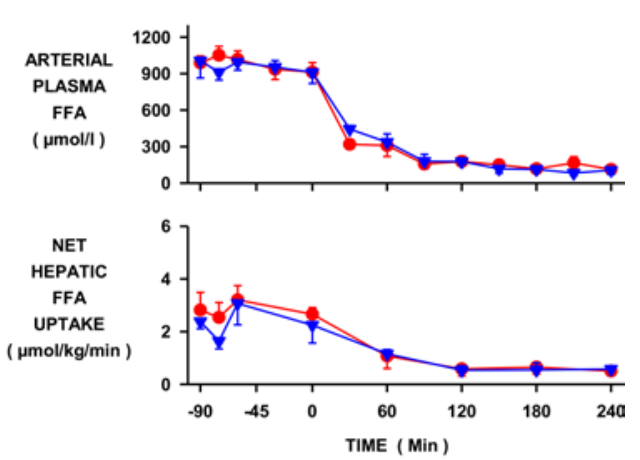

$\mathbf{F}$
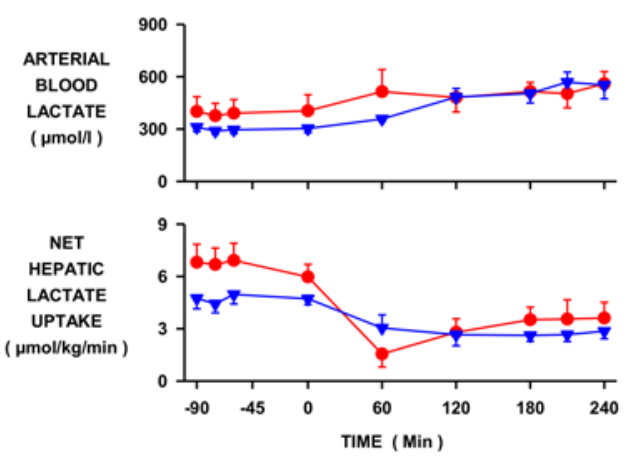

H
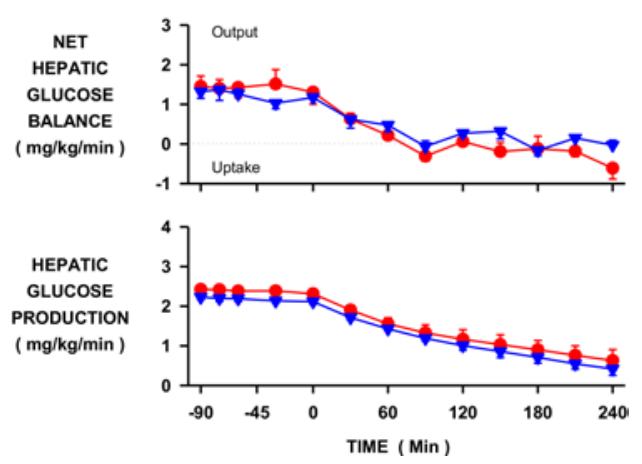

Figure 2. Effect of a fall in glucagon on hepatic glucose production during hyperinsulinemia. Insulin was elevated 6 -fold by portal vein insulin infusion in conscious dogs in the INS control $(n=6)$ and INS+GGN glucagon-clamp $(n=5)$ groups. Glucagon was infused into the portal vein in the latter to prevent a decrease in its level at the liver. (A) Arterial and hepatic sinusoidal plasma insulin levels. (B) Arterial plasma glucose levels and peripheral glucose infusion rate. (C) Arterial plasma C-peptide and hepatic sinusoidal plasma glucagon levels. (D) Arterial plasma free fatty acid (FFA) levels and net hepatic FFA uptake. (E) Arterial blood glycerol levels and net glycerol hepatic uptake. (F) Arterial blood lactate levels and net hepatic lactate uptake. (C) Intrahepatic gluconeogenic and glycogenolytic fluxes. (H) Net hepatic glucose balance and hepatic glucose production. Values are means \pm SEM. *Denotes a difference $(P<$ 0.05 ; 2-way repeated measure ANOVA) between groups.

was not reduced and the greater substrate supply allowed for increased net hepatic glycogen synthesis (Figure 1G). Despite the effects of FFA on intrahepatic carbon fluxes, the fall in FFA had no impact on the regulation of HGP. Net hepatic glucose balance and tracer-determined HGP were suppressed over the same time course and to the same extent regardless of the arterial FFA concentrations (Figure $1 \mathrm{H}$ ).

Glucagon-clamp group versus control. Arterial plasma glucose levels, glucose infusion rates, and whole-body glucose utilization did not differ between the INS (con-

trol) and glucagon-clamp (INS+GGN) groups (Figure 2B and Table 1). Hepatic sinusoidal glucagon levels declined in the INS group ( $49 \pm 5$ to $40 \pm 4 \mathrm{pg} / \mathrm{ml}$; basal vs. experimental periods, respectively). This decrease may have been larger than it appears since $\sim 25-30 \mathrm{pg} / \mathrm{ml}$ of what is measured by the Millipore glucagon RIA is not glucagon (nonspecific cross-reacting material, unpublished results from the Vanderbilt University Hormone Assay and Analytical Services Core). Glucagon levels were maintained at basal (47 
Table 1. Tracer-determined whole-body glucose utilization

\begin{tabular}{l|c|c|c|c|c|c|}
\hline & $\begin{array}{c}\text { Basal } \\
\text { Period }\end{array}$ & \multicolumn{5}{|c}{ Experimental Period (minutes) } \\
& $2.4 \pm 0.1$ & $\mathbf{6 0}$ & $\mathbf{1 2 0}$ & $\mathbf{1 8 0}$ & $\mathbf{2 1 0}$ & $\mathbf{2 4 0}$ \\
\hline INS & $2.2 \pm 0.1$ & $6.9 \pm 0.8$ & $7.8 \pm 1.3$ & $8.6 \pm 1.4$ & $9.1 \pm 1.3$ & $9.7 \pm 1.4$ \\
INS+FFA & $2.2 \pm 0.1$ & $6.8 \pm 0.5$ & $9.4 \pm 0.5$ & $7.3 \pm 0.6$ & $7.5 \pm 0.5$ & $7.8 \pm 0.6$ \\
INS+GGN & $2.2 \pm 0.0$ & $5.9 \pm 0.5$ & $7.3 \pm 0.4$ & $8.1 \pm 0.9$ & $9.9 \pm 1.0$ & $10.0 \pm 0.9$ \\
INS-BRAIN & $2.3 \pm 0.0$ & $7.0 \pm 0.6$ & $7.4 \pm 0.7$ & $7.5 \pm 0.9$ & $7.9 \pm 0.7$ & $9.1 \pm 0.7$ \\
INS-COMPLETE & & & 7.9 & 7.0 & $7.6 \pm 0.9$
\end{tabular}

Insulin was elevated 6-fold by portal vein insulin infusion in conscious dogs. Either the direct and indirect effects of insulin on the liver were all present (INS; $n=6$ ), or plasma FFAs were clamped at basal (INS+FFA; $n=5$ ), plasma glucagon was clamped at basal (INS+GGN; $n=5$ ), increased brain insulin signaling was blocked (INS-BRAIN; $n=9$ ), or all of the indirect effects of insulin were blocked (INS-COMPLETE; $n=5$ ). Values are mean \pm SEM.

\pm 6 to $45 \pm 5 \mathrm{pg} / \mathrm{ml}$ ) in the INS+GGN group due to the intraportal infusion of glucagon (Figure 2C). Arterial plasma FFA and blood glycerol levels were reduced similarly in both groups, as were net hepatic uptakes of FFA and glycerol (Figure 2, D and E). Net hepatic lactate uptake was reduced and arterial lactate levels increased similarly in both groups (Figure 2F). Intrahepatic gluconeogenic and glycogenolytic fluxes responded similarly whether or not glucagon levels decreased, although at the last time point net hepatic glycogen synthesis was modestly lower in the INS+GGN group (Figure 2G). The decrease in hepatic glucagon levels caused by hyperinsulinemia had no significant effect on the time course or extent of suppression of net glucose balance or HGP (Figure $2 \mathrm{H}$ ).

Brain insulin signaling block group versus control. Effective blockade of brain insulin signaling is important to the interpretation of our data. Previous rodent and dog studies have shown that hepatic signal transducer and activator of transcription-3 (STAT3) phosphorylation results from brain insulin action $(22,40-42)$. In addition, suppression of HGP by the brain-liver insulin axis requires a neurally mediated increase in hepatic STAT3 phosphorylation (40). In those studies, intracerebroventricular (i.c.v.) infusion of a PI3-kinase inhibitor, $\mathrm{K}_{\text {ATP }}$ channel inhibitor, or hepatic denervation effectively prevented brain insulin-mediated increases in liver p-STAT3, which in turn completely blocked downstream effects at the liver, including changes in mRNA and protein levels (glucokinase and multiple gluconeogenic and glycogenolytic enzymes) $(22,42)$. Thus, prevention of STAT3 phosphorylation indicates successful blockade of the brain-liver insulin axis. Infusion (i.c.v.) of the inhibitors was initiated 60 minutes prior to the start of hyperinsulinemia in the present study since in previous studies we showed that the full blockade of brain insulin action occurs within 1 hour (22).

As expected, brain insulin signaling increased hepatic STAT3 phosphorylation during hyperinsulinemia, whereas i.c.v. infusion of a PI3-kinase inhibitor (LY294002) or insulin receptor antagonist (S961) eliminated that downstream effect (Figure 5). This indicates that the brain-liver insulin axis was activated in the INS group but not in the brain insulin-block (INS-BRAIN) group (Figure 5). Although LY294002 was previously shown to prevent activation of the brain-liver insulin axis in the rat and $\operatorname{dog}(17,22,42)$, we infused S961 into the third ventricle in a subgroup in order to determine whether inhibition of all brain insulin signaling pathways (including MAP-kinase) would confirm the data from the PI3-kinase inhibitor studies. As occurred with i.c.v. infusion of LY294002, S961 prevented hyperinsulinemia from increasing hepatic STAT3 phosphorylation relative to the INS group $(1.18 \pm 0.10$ and $1.18 \pm 0.15$ in the LY and S961 groups, respectively, vs. $1.91 \pm 0.25$ in the INS group). This suggests that brain insulin action acts primarily through PI3-kinase, rather than MAP-kinase, in agreement with what was previously reported (17). There were no significant differences between the results in the LY294002 and S961 groups with regard to changes in insulin or glucagon secretion, lipolysis, net hepatic glucose balance, endogenous glucose production, or net hepatic glycogenolytic or gluconeogenic fluxes; therefore, the data from the 2 groups were combined.

Arterial plasma glucose levels, glucose infusion rates, and whole-body glucose utilization did not differ between the INS and INS-BRAIN groups (Figure 3B and Table 1). Hepatic sinusoidal glucagon levels were suppressed similarly in both groups (Figure 3C), as were arterial plasma FFA and glycerol levels and net hepatic FFA and glycerol uptake (Figure 3, D and E). Net hepatic lactate uptake fell similarly in both groups, in both cases causing a rise in arterial lactate levels (Figure 3F). Intrahepatic gluconeogenic and glycogenolytic fluxes did not differ between groups (Figure 3G). The increase in brain insulin signaling caused 

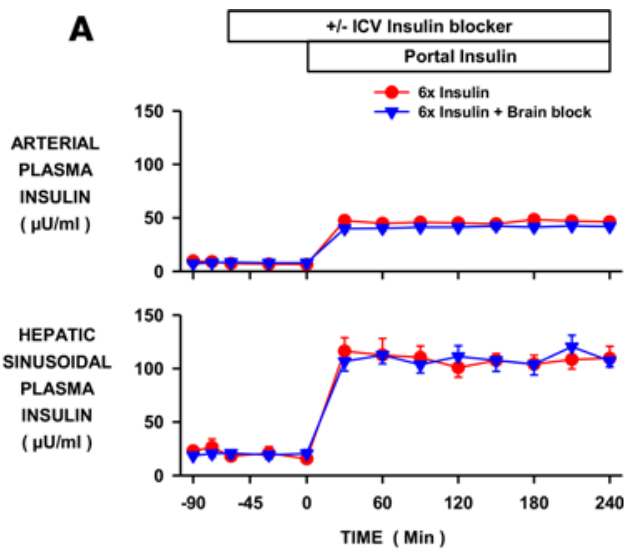

\section{C}
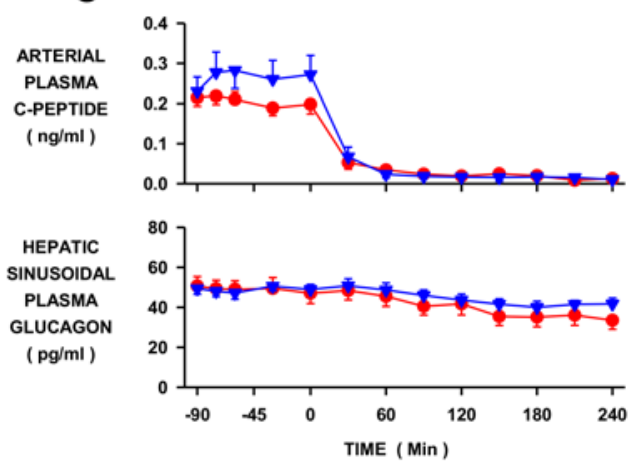

E
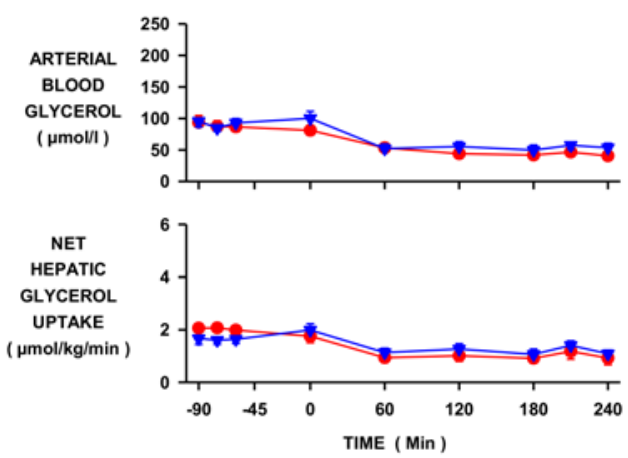

G
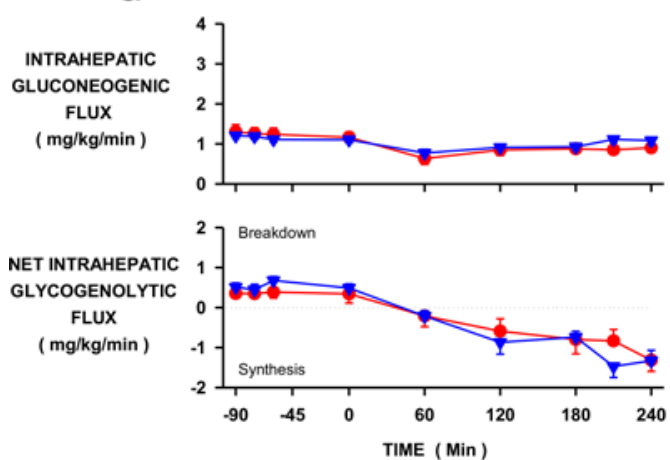
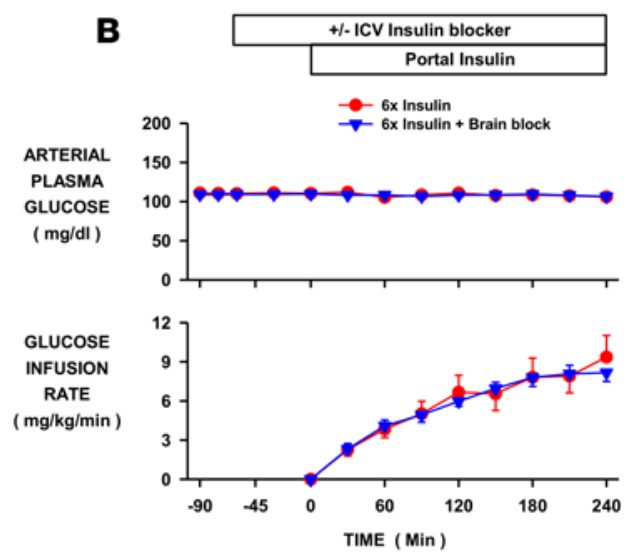

D
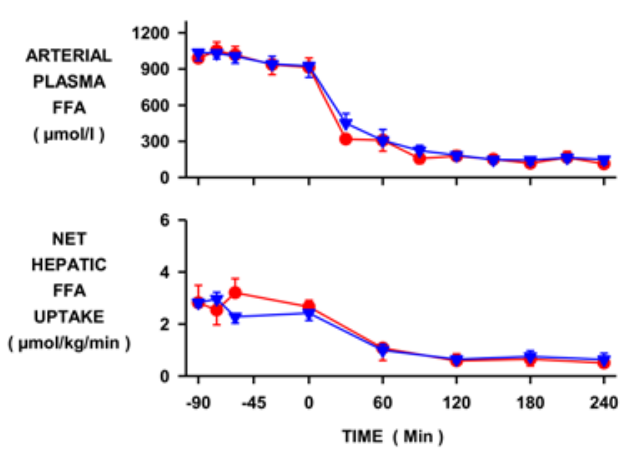

F
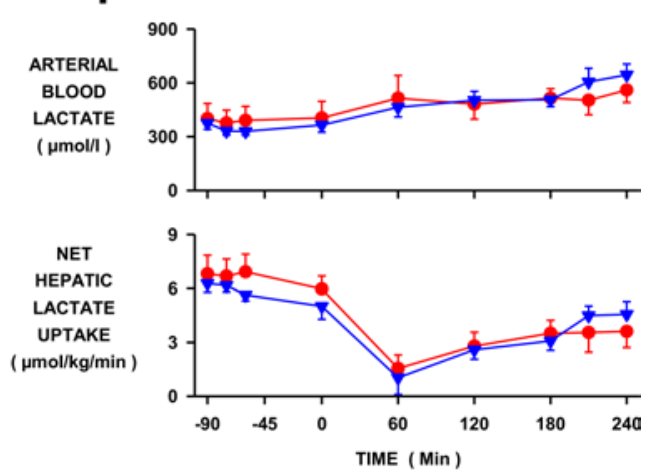

\section{H}
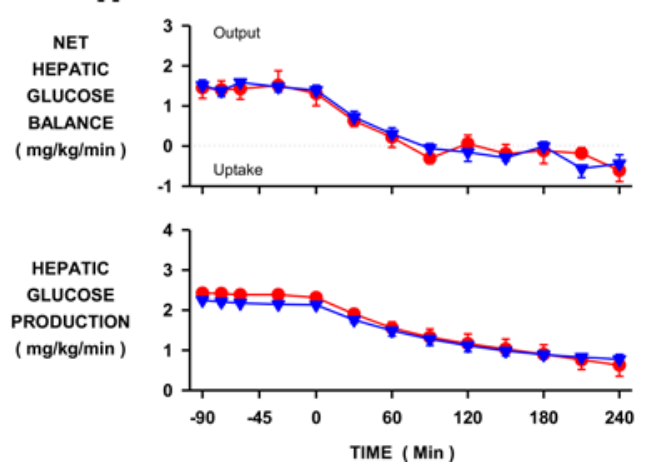

Figure 3. Effect of increased brain insulin signaling on hepatic glucose production during hyperinsulinemia. Insulin was elevated 6-fold by portal vein insulin infusion in conscious dogs in the INS control $(n=6)$ and brain insulin-block (INS-BRAIN, $n=9$ ) groups. In the latter, a PI3-kinase inhibitor or insulin receptor antagonist was infused into the third ventricle to block an increase in brain insulin signaling. (A) Arterial and hepatic sinusoidal plasma insulin levels. (B) Arterial plasma glucose levels and peripheral glucose infusion rate. (C) Arterial plasma C-peptide and hepatic sinusoidal plasma glucagon levels. (D) Arterial plasma free fatty acid (FFA) levels and net hepatic FFA uptake. (E) Arterial blood glycerol levels and net glycerol hepatic uptake. (F) Arterial blood lactate levels and net hepatic lactate uptake. (G) Intrahepatic gluconeogenic and glycogenolytic fluxes. (H) Net hepatic glucose balance and hepatic glucose production. Values are means \pm SEM. ICV, intracerebroventricular.

by hyperinsulinemia had no effect on the time course or extent to which inhibition of net hepatic glucose balance or HGP occurred (Figure 1H).

Complete indirect block group versus control. Intralipid and heparin, glucagon, and brain insulin signaling inhibitors were infused in the complete indirect insulin-block (INS-COMPLETE) group to simultaneously block all of insulin's indirect effects on HGP. Infusion (i.c.v.) of LY294002 and S961 prevented the increase in
$5)$. Arterial plasma glucose brain-liver insulin signaling that occurred in the INS (control) group (Figure 5). Arterial plasma glucose did not differ between groups and the tendency for glucose infusion rates to diverge at the end of the clamp is explained by the impact of FFA on whole-body glucose utilization rates (Figure 4B and Table 1). Unlike the INS group, hepatic sinusoidal glucagon levels rose modestly in the INS-COMPLETE group (42 \pm 5 to $62 \pm 8 \mathrm{pg} / \mathrm{ml}$; basal period vs. last hour of the experimental period, respectively; 
A

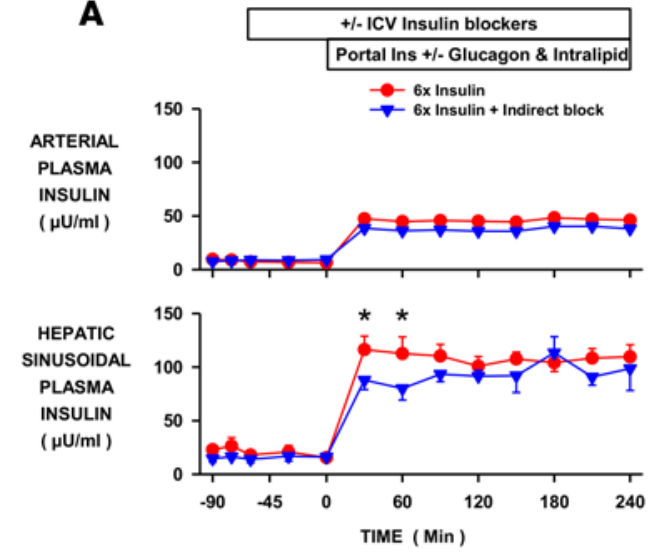

C

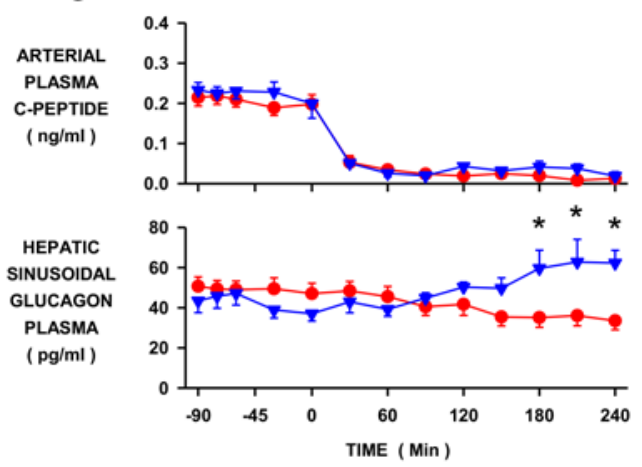

E

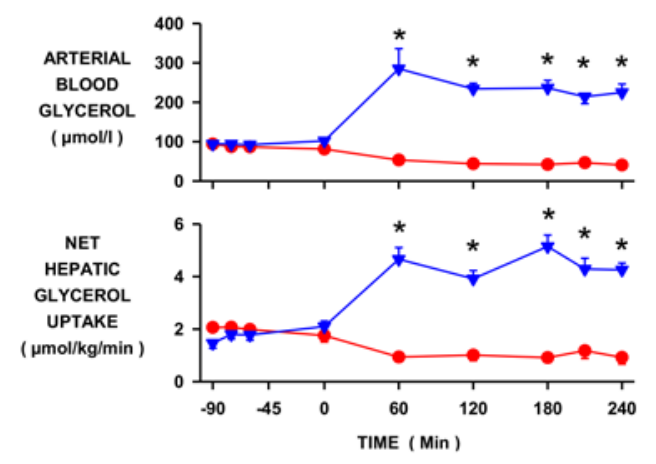

G
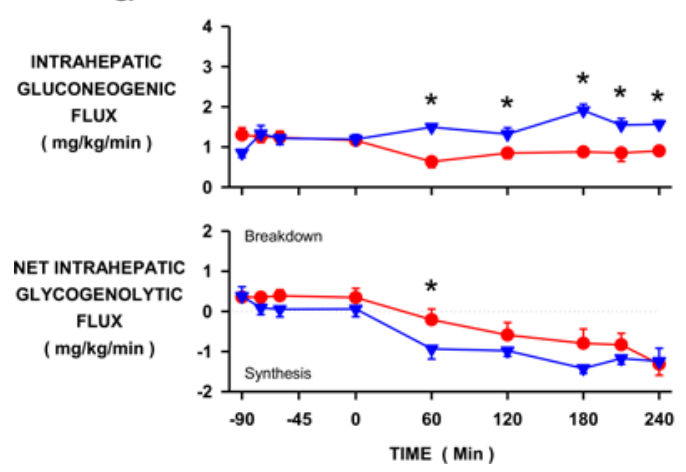

B
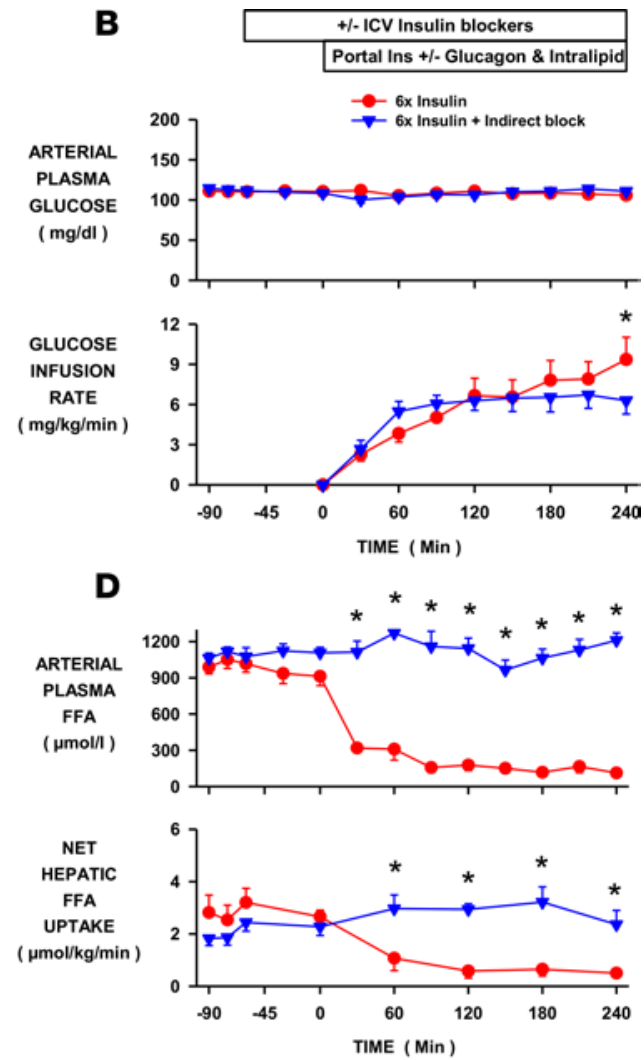

\section{$\mathbf{F}$}
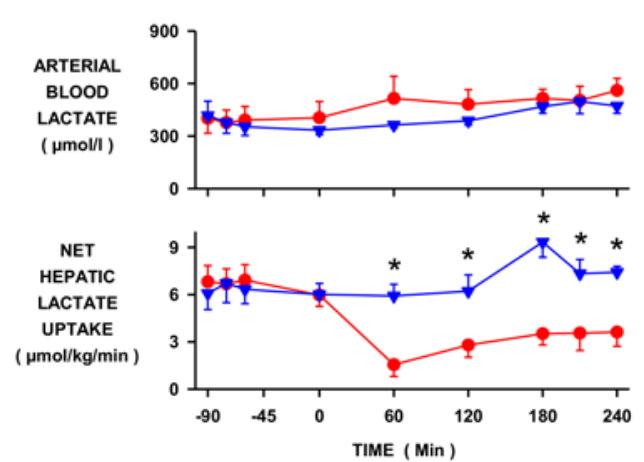

H
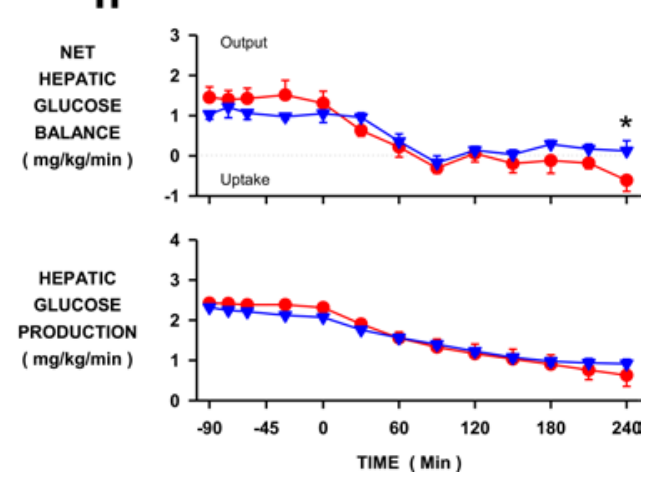

Figure 4. Effect of blocking all of the indirect effects of insulin on hepatic glucose production during hyperinsulinemia. Insulin was elevated 6 -fold by portal vein insulin infusion in conscious dogs in the INS control ( $n$ $=6)$ and INS-COMPLETE ( $n$ $=5)$ groups. All of insulin's indirect effects were blocked in the latter with infusions of intravenous intralipid, portal vein glucagon, and third ventricle infusion of LY294002 and S961.

(A) Arterial and hepatic sinusoidal plasma insulin levels. (B) Arterial plasma glucose levels and peripheral glucose infusion rate. (C) Arterial plasma C-peptide and hepatic sinusoidal plasma glucagon levels. (D) Arterial plasma free fatty acid (FFA) levels and net hepatic FFA uptake. (E) Arterial blood glycerol levels and net glycerol hepatic uptake. (F) Arterial blood lactate levels and net hepatic lactate uptake. (G) Intrahepatic gluconeogenic and glycogenolytic fluxes.

(H) Net hepatic glucose balance and hepatic glucose production. Values are means \pm SEM. ${ }^{*}$ Denotes a difference $(P<0.05 ; 2$-way repeated measure ANOVA) between groups. ICV, intracerebroventricular.

Figure 4C). Arterial FFA and glycerol levels and net hepatic FFA and glycerol uptake were rapidly reduced by hyperinsulinemia in INS but remained at basal values or were elevated in INS-COMPLETE (Figure 4, D and E). Similarly, net hepatic lactate uptake was reduced in INS (Figure 4F) but did not decrease when the arterial FFA levels were clamped at baseline values. Whereas gluconeogenic flux to G6P was reduced by hyperinsulinemia and the associated fall in FFA in INS, there was no decrease in gluconeogenic flux in INS-COMPLETE, and sustained flux into intrahepatic G6P provided the necessary substrate for increased net hepatic glycogen synthesis (Figure 4G). Although net hepatic glucose uptake was modestly greater in INS compared with INS-COMPLETE at the end of 


\section{Liver \\ P-STAT3 / Cyclophilin B}
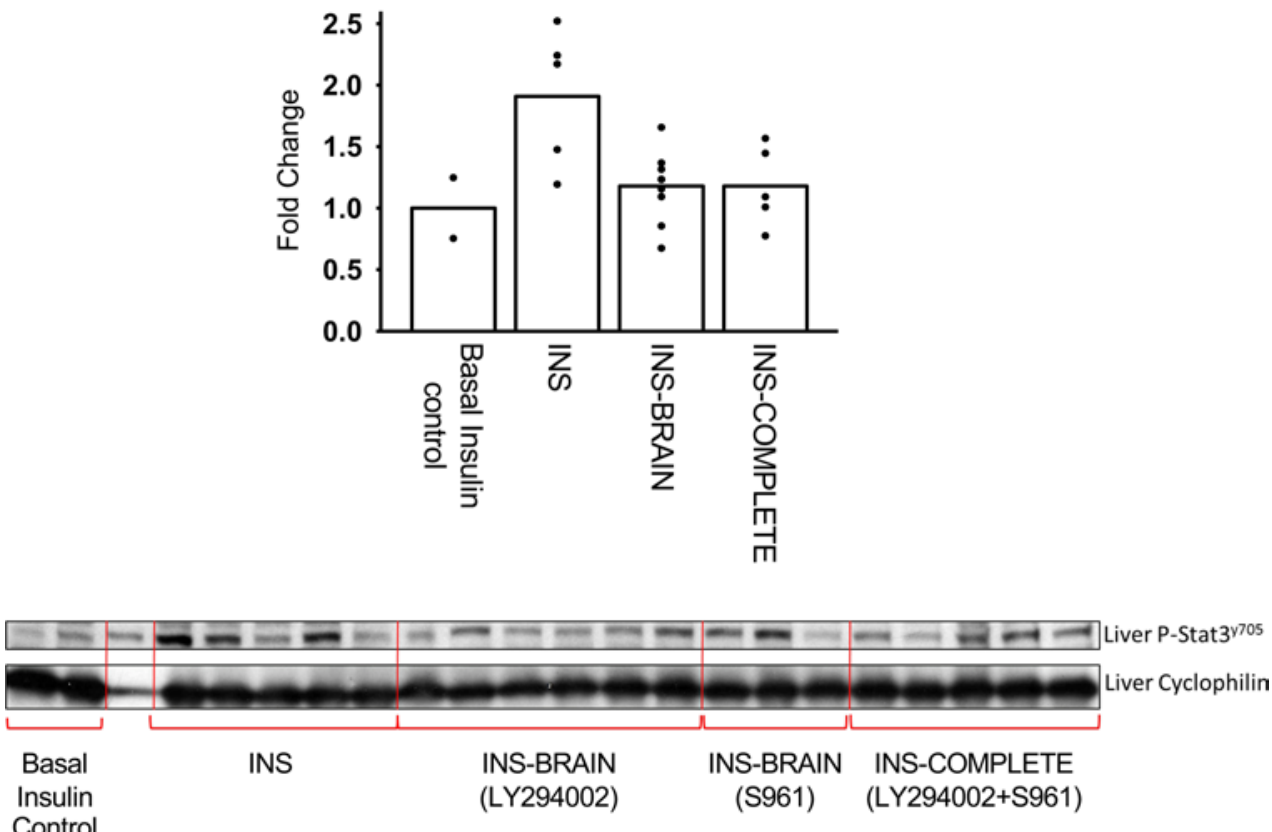

Control
Figure 5. CNS activation of STAT-3 phosphorylation (Tyr705) in the liver. Results are expressed relative to liver from overnight-fasted control dogs in which insulin was basal ( $n$ $=2$ ) compared to when insulin was elevated 6 -fold by portal vein insulin infusion in the INS control $(n=5)$, brain insulin-block (INS-BRAIN, $n=9$ ), and INS-COMPLETE (all of insulin's indirect effects blocked, $n=$ 5) groups. Values are means \pm SEM. ${ }^{*}$ STAT-3 phosphorylation in the INS BRAIN and INS-COMPLETE groups was reduced $(P<0.05)$ compared with the INS group.

the study, probably due to the mismatch in hepatic glucagon levels, the indirect effects of insulin on lipolysis, the $\alpha$ cell, and the brain were not required for the rapid suppression of net hepatic glucose balance or HGP caused by portal vein insulin delivery (Figure 4H).

\section{Discussion}

Glucose production is dysregulated in individuals with diabetes and as such it is a primary contributor to hyperglycemia. Understanding how insulin normally regulates HGP as well as the defect occurring in diabetes is crucial for the development of effective therapies targeting this process. It is well established that insulin can independently regulate HGP by both its direct or indirect hepatic effects $(5,43)$, yet considerable controversy remains regarding the relative importance of each mechanism. The central effects of insulin have been said to be required, necessary, crucial, or even essential for the suppression of HGP by insulin $(17,18,44-49)$, and recently it was concluded that suppression of lipolysis is the major mechanism by which insulin inhibits HGP (11). To date, both the direct and indirect effects of insulin have been identified as being the dominant signal by which a rise in insulin reduces HGP in vivo $(1,50)$, leaving the issue unresolved. The present study seeks to present a unifying hypothesis, by showing that the context under which insulin acts is a primary determinant of its mechanism of action. By extending observations made in the rodent to a large animal model we hope to facilitate the translation of relevant findings to the human.

Previously we investigated insulin's indirect effects on HGP in the dog (i.e., the effect of a selective increase in peripheral insulin levels while insulin at the liver remained at basal). Insulin did indeed eventually inhibit HGP indirectly (within 60 minutes vs. 15 minutes with portal insulin infusion) (5), primarily due to a fall in plasma FFAs (13). In addition, hyperinsulinemia suppresses glucagon secretion over time, and basal insulin inhibits HGP in the absence of glucagon $(15,21)$. Furthermore, when brain insulin action was selectively increased by insulin infusion either directly into the brain (i.c.v.), or into the blood perfusing the brain (carotid and vertebral arteries), CNS signaling affected multiple downstream molecular targets in the liver (transcription, translation, and phosphorylation) but it did not affect HGP (22). On the other hand, net hepatic glucose uptake increased modestly after a several-hour delay (22). These studies and those performed by others establish the existence of insulin's indirect effects, but they do not address their relevance under physiologic conditions.

Therefore, the purpose of this study was to definitively determine the relative contribution of insulin's direct and indirect effects to the inhibition of HGP in the context of a physiologic increase in insulin secretion. To the best of our knowledge, this study is the first to simultaneously eliminate all of insulin's indirect effects on HGP during a physiologic rise in the portal vein insulin level. It was important to concurrently eliminate all of the indirect mechanisms of control because it is possible that they could compensate for one another when blocked individually. We found that HGP was suppressed to the same extent, and with 
the same time course, in response to increased portal vein insulin delivery regardless of whether or not insulin's indirect effects on adipose tissue, pancreatic $\alpha$ cells, or the brain were intact. Our data do not dispute the existence of a brain-liver insulin axis, or the ability of a suppression of lipolysis or glucagon secretion to independently regulate HGP, but they underscore their redundancy in times of physiologic hyperinsulinemia. The present data clearly demonstrate that the indirect effects of insulin are not required for the suppression of HGP when insulin secretion increases and that it is the hormone's direct effect on the liver that provides the dominant inhibitory signal.

Superficially, these results are in disagreement with other findings, but experimental context is crucial. Recently, Perry et al. (11) concluded from studies performed in overnight-fasted rats that the major mechanism by which insulin suppresses HGP is through the suppression of lipolysis, leading to a decrease in gluconeogenesis. It is important to note that the livers of overnight-fasted rats are almost completely glycogen depleted (33), entirely gluconeogenic (11), and therefore have no capacity for the inhibition of glycogenolysis by insulin. In contrast, fed rodents and fasted large animals respond to insulin by rapidly suppressing glycogenolysis (2, 7-9). In addition, Perry et al. infused insulin into a peripheral vein at a rate ( $3 \mathrm{mU} / \mathrm{kg} / \mathrm{min}$ ) that has been shown to increase arterial levels 3-fold $(17,18,51)$, but which would not have elevated insulin at the liver (26). Therefore, that study does not provide insight into the suppression of HGP due to inhibition of lipolysis in the context of an increase in insulin secretion (i.e., a rise in portal vein insulin delivery). Instead, like many previous studies, those results confirm that the suppression of HGP by insulin's indirect action is primarily due to the hormone's effect on lipolysis $(10,12-14)$. In contrast, the present study demonstrates that the suppression of lipolysis provides no further suppression of HGP in the presence of insulin's direct effect.

Because the direct and indirect mechanisms by which insulin regulates HGP are distinct and independent, their combined effects could be additive or even synergistic. Insulin directly inhibits HGP via rapid effects on glycogen metabolism, whereas inhibition of lipolysis drives an allosterically mediated reduction in gluconeogenesis (11). Of note, the signaling events that are downstream of hepatocyte insulin receptor activation are unchanged by the suppression of lipolysis and the allosteric effects of acetyl CoA. For example, insulin's inhibitory effect on glycogen breakdown through PI3-kinase is present whether or not the indirect effects of insulin are operative (42). It is notable that clamping plasma FFAs at basal levels during hyperinsulinemia in the present study prevented suppression of intrahepatic gluconeogenic flux. This was likely due to sustained hepatic acetyl CoA levels, which would have maintained flux through pyruvate carboxylase, as shown by Perry et al. (11). Indeed, net hepatic lactate uptake persisted in the presence of FFAs and this provided greater substrate for flux into glycogen. Despite preventing a decrease in intrahepatic gluconeogenic flux, blockade of insulin's lipolytic effect did not limit the ability of insulin to reduce HGP, because the effect was offset by an equal increase in glycogen synthesis. Thus, it was insulin's overriding direct effect on glycogen metabolism that determined HGP.

Obici et al. (17) concluded that the brain-liver insulin axis involves insulin signaling through PI3-kinase, not MAP-kinase, since i.c.v. administration of PI3-kinase inhibitors (LY294002 and wortmannin) blocked the suppression of glucose production by CNS insulin, whereas an inhibitor of MAP-kinase did not. On the other hand, Filippi et al. (52) found that central insulin action could inhibit glucose production by acting via either PI3-kinase in the hypothalamus or MAP-kinases (Erk1/2) in the dorsal vagal complex. In either case, the neuronal PI3-kinase and MAP-kinase pathways were found to converge downstream, requiring the activation of $\mathrm{K}_{\text {ATP }}$ channels $(18,52)$, which would then cause vagally mediated phosphorylation of hepatic STAT3 $(22,40-42)$, and suppression of HGP. In the present study, the lack of increase in hepatic p-STAT3 in the group treated with LY294002 demonstrates that brain-liver insulin signaling was completely blocked by the PI3-kinase inhibitor. In addition, there was no difference in the effects at the liver whether signaling through PI3-kinase alone or all brain insulin receptor signaling was blocked (LY294002 vs. S961). Finally, we previously found that the liver markers of brain insulin action were equivalently blocked by i.c.v. administration of LY294002 or a $\mathrm{K}_{\text {ATP }}$ channel inhibitor (42). Thus, like Obici et al. (17), we found that the brain-liver insulin axis requires the activation of neuronal PI3-kinase, not MAP-kinase.

In addition to CNS-liver neural input $(17,18)$, brain insulin action has the potential to alter HGP through a variety of mechanisms. For example, besides insulin's direct effects on pancreatic hormone secretion $(53,54)$ and adipose tissue lipolysis $(37)$, brain insulin action may neurally lower plasma glucagon (55) and FFA $(41,49,56)$ and perhaps feed-forward to increase insulin secretion $(57,58)$, thereby indirectly inhibiting HGP. Caution must be applied when interpreting the results from studies in which insulin is 
injected directly into the brain, is administered intranasally to selectively increase brain insulin concentrations, or when brain insulin action is blocked during a peripheral insulin clamp in which the arterial but not hepatic insulin levels are elevated $(17,18,22,59)$. While such studies establish the potential for CNS insulin to influence HGP, lipolysis, or pancreatic hormone secretion, they do not address the physiologic impact of those mechanisms since insulin levels always increase proportionally, throughout the body, when insulin is secreted endogenously.

Brain insulin action had no effect on lipolysis during hyperinsulinemia, since glycerol and FFA levels did not differ when CNS insulin signaling was blocked. This demonstrates that the lipolytic and re-esterification responses were determined by insulin's noncentral effects when insulin was elevated simultaneously at both adipose tissue and the brain. Thus, the previously demonstrated CNS effect of insulin on lipolysis $(41,49,56)$ is redundant to insulin's acute direct adipose tissue effects, although brain insulin may regulate lipolysis under other circumstances.

The autonomic nervous system regulates the pancreatic $\alpha$ cell, and hypothalamic insulin action has been shown to suppress fasting glucagon secretion in the rodent (55). It is noteworthy that despite the same rates of glucagon replacement in the glucagon-clamp and complete indirect-block groups, plasma glucagon concentrations were modestly greater in the latter. Thus, it is possible that blocking brain insulin action interfered with the suppression of glucagon secretion, but only in the presence of basal FFAs, which are known stimulators of glucagon secretion (60). Regardless, suppression of glucagon secretion was not required for the full inhibition of HGP in the context of increased insulin secretion.

Although exogenous insulin infusion has been shown to suppress insulin secretion in vivo, the direct effect of insulin on the $\beta$ cell has been called into question (34-36). In addition, while it has been shown that insulin secretion can be neurally controlled, the role of brain insulin action in causing this effect is uncertain (36). A pharmacologic dose of insulin injected into the lateral cerebral ventricle was shown to increase insulin secretion in dogs (57) and brain $\mathrm{K}_{\text {ATP }}$ channel activation caused a similar effect in rats (58). Thus, brain insulin action may feed-forward to stimulate insulin secretion and thereby inhibit HGP. In contrast, others have found that insulin can inhibit its own secretion through a neural mechanism, without acting directly on the $\beta$ cell (61). In the present study, however, exogenous insulin rapidly and completely suppressed basal insulin secretion through an effect that was not driven by CNS insulin action, because it occurred even when brain insulin signaling was blocked. Likewise, although FFAs drive $\beta$ cell secretion (62), the inhibition of insulin secretion by exogenous insulin did not depend on the suppression of lipolysis since the same reduction of C-peptide occurred even when FFAs were clamped at basal levels.

This study demonstrates that insulin's direct effect on the liver was sufficient to elicit hyperinsulinemia's full, time-dependent response. In contrast, previous studies indicate that although insulin can indirectly suppress HGP in the absence of a rise in the hepatic insulin level, the response is imperfect, at least with regard to timing and perhaps to degree. While HGP is rapidly suppressed by its direct effect $(<15$ minutes; see refs. 3,5$)$, its indirect effects are delayed $(5,13)$. This is not surprising since, unlike the open fenestrations of liver sinusoids, which allow for rapid movement of insulin from the blood to its hepatocyte receptor, endothelial cell tight junctions in adipose tissue slow insulin translocation, causing a lag in the suppression of lipolysis. As a result, it takes time for plasma FFA levels to fall (5, 13). Likewise, the insulin-induced fall in plasma glucagon is modest and requires at least an hour to be seen. The brain-liver insulin axis is even slower $(17,18,22,59)$, since several hours of hyperinsulinemia are prerequisite for an increase in hepatic STAT3 phosphorylation to occur $(9,40)$, and then there is the additional time required for changes in gluconeogenic gene transcription and translation to affect enzyme activity (9). Thus, the rapid suppression HGP caused by a rise in portal vein insulin occurs prior to the impact of insulin's indirect effects.

The present study dealt with the suppression of HGP under euglycemic conditions, but insulin normally rises in tandem with glucose during meal ingestion. This raises the question of whether the indirect effects of insulin might play a greater role in the regulation of hepatic glucose metabolism during feeding. For example, feeding affects neural networks (63) that might amplify the effects of brain insulin on the liver. On the other hand, insulin's effects on HGP via suppression of lipolysis and glucagon secretion would be offset if plasma FFA and glucagon levels rise in response to feeding. In addition, hyperglycemia itself suppresses HGP, even under basal insulin conditions (64); therefore, the inhibition of HGP by combined hyperinsulinemia and hyperglycemia is even more rapid and pronounced than with hyperinsulinemia alone, with the liver switching from a state of glucose production to storage in minutes. Thus, one could 
argue that euglycemic conditions may actually favor the detection of insulin's indirect effects. It is worth noting that chronic alterations in the mediators of those effects (NEFA, glucagon, and/or neural signaling to the liver) could influence how HGP is acutely regulated. For example, hyperlipidemia leading to liver fat accumulation causes hepatic insulin resistance. Carefully controlled experiments will be required to identify the manner in which hepatic insulin tone is set and to determine how feeding signals may impact the various mechanisms by which insulin regulates liver glucose production and uptake.

At present, most insulin-dependent patients receive insulin through the peripheral (i.e., subcutaneous) route. The resulting over-insulinization of nonhepatic tissues and under-insulinization of the liver can contribute to metabolic dysfunction, including insulin resistance, coagulation abnormalities, weight gain, and alterations in body fat distribution and lipid metabolism, and the imbalance in insulin gradient is also a risk factor for hypoglycemia, hypertension, atherosclerosis, and long-term micro- and macrovascular complications (25). Thus, there are important therapeutic implications to understanding how insulin secretion regulates HGP, as well as to knowing whether insulin's indirect actions are less effective in patients with diabetes who are treated with insulin peripherally. Metabolic dysfunction may be avoided by selectively targeting the liver's direct effect, for example with oral or hepato-preferential insulin analogs, or with intraperitoneal insulin delivery (25).

In summary, while it is abundantly clear that insulin can regulate HGP through multiple means, experimental context is a major determinant of the importance of each mechanism. In the physiologic setting, portal vein insulin delivery rapidly suppresses HGP through its direct effects on the liver in healthy animals. The indirect effects of insulin, while present, are redundant and without detectable impact. Likewise, the inhibition of lipolysis and glucagon and insulin secretion, in response to portal vein insulin delivery, do not require insulin's indirect actions, but are driven by its direct effects on the adipocyte, $\alpha$ cell, and $\beta$ cell, respectively.

\section{Methods}

Animal care and surgical procedures. Experiments were performed using conscious 42-hour-fasted dogs of either sex (19-24 kg) as previously described (42). This length of fast was used to increase the likelihood that brain insulin action would produce a metabolic effect on the liver (greater potential for suppression of gluconeogenesis following a longer fast). It should be noted that a 42-hour fast in the dog does not induce hypoglycemia, raise the plasma levels of stress hormones, or eliminate liver glycogen. Arterial cortisol or catecholamine levels did not differ over time or between groups (data not shown).

Seventeen days prior to the study, intraportal infusion catheters were implanted in the jejunal and splenic veins, blood sampling catheters were inserted in the femoral artery, hepatic portal vein, and hepatic vein, and ultrasonic blood flow probes were placed around the hepatic artery and portal vein, as previously described $(8,22)$. Ten days prior to the study, stereotaxic i.c.v. cannulation was performed as previously described (22). All dogs were healthy, as indicated by a return to presurgical food intake and body weight, leukocyte count less than $18,000 / \mathrm{mm}^{3}$, hematocrit greater than $35 \%$, and normal stools.

Experimental design. Experiments consisted of equilibration ( -180 to -90 minutes), basal ( -90 to 0 minutes), and experimental (0-240 minutes) periods. At -180 minutes, $\left[3-{ }^{3} \mathrm{H}\right]$ glucose administration was started (35 $\mu \mathrm{Ci}$ priming dose; $0.35 \mu \mathrm{Ci} / \mathrm{min}$ constant infusion). Intraportal insulin infusion $(1.8 \mathrm{mU} / \mathrm{kg} /$ min) was initiated at 0 minutes to bring about a 6-fold rise in insulin in the liver and nonhepatic tissues and glucose was infused into a peripheral vein to maintain euglycemia. At the end of each experiment the animals were administered a lethal dose of pentobarbital and liver and brain tissues were rapidly harvested, freeze-clamped, and stored at $-70^{\circ} \mathrm{C}$.

There were 5 hyperinsulinemic/euglycemic groups: a control group (INS; $n=6$ ); a fat-clamp group (INS+FFA; $n=5$ ) in which intravenous intralipid and heparin were infused to maintain arterial FFA levels at a basal value throughout the experimental period of the study; a glucagon-clamp group (INS+GGN; $n=5$ ) in which glucagon was infused into the portal vein to maintain glucagon at a basal level; a brain insulin-block group (INS-BRAIN; $n=9$ ) in which a PI3-kinase inhibitor (LY294002; $n=6$; Sigma-Aldrich) or insulin receptor antagonist (S961; $n=3$; gift from Novo Nordisk) was infused into the third ventricle to block increased brain insulin signaling; or a group in which a block of all 3 of insulin's indirect effects was brought about (INS-COMPLETE; $n=5$; intravenous intralipid, portal vein glucagon, and third ventricle LY294002 and S961 were infused). Intraportal glucagon was infused in the INS-GGN and INS-COMPLETE groups beginning at 0 minutes and increasing at 30-minute intervals using the following algorithm: $0.01,0.09,0.15,0.27,0.33,0.38,0.43$, and $0.49 \mathrm{ng} / \mathrm{kg} / \mathrm{min}$, 
respectively. LY294002 (3.82 ng/kg/min, $0.01 \mathrm{ml} / \mathrm{min})$ and/or S961 (25.2 ng/kg/min; $0.01 \mathrm{ml} / \mathrm{min})$ dissolved in artificial cerebrospinal fluid were infused as previously described to completely block brain insulin action $(22,42)$ beginning at -60 minutes. The latter was chosen to provide sufficient time for the insulin signaling blockers to act as shown in our earlier study (22).

Biochemical analysis. Hepatic STAT3 phosphorylation was assayed using standard Western blot procedures as previously described $(9,22)$. Liver tissue from 42-hour-fasted untreated animals (basal insulin/ glucose) was used to provide reference values so as to allow for the calculation of changes in molecular markers of insulin signaling.

Metabolic analysis. The plasma insulin and glucagon levels entering the liver sinusoids were calculated by summing arterial and portal vein insulin levels after multiplying each respectively by the percentage contributions of hepatic artery $(\sim 20 \%)$ and portal vein $(\sim 80 \%)$ blood flow to total hepatic blood flow in each study. We assessed glucose flux by measuring net hepatic glucose balance (the sum of hepatic glucose output and uptake) and its components: the net rate of G6P formation from the glycolytic/gluconeogenic pathways and from the glycogen synthetic/glycogenolytic pathways, as previously described $(8,22)$. Tracerdetermined glucose turnover was calculated as previously described $(8,22)$. Glucagon was measured using the EMD Millipore GL-32K RIA. Other hormone and substrate concentrations were determined using standard procedures as reported previously $(8,22)$.

Statistics. Metabolic data were analyzed using 2-way repeated-measures ANOVA (group $\times$ time) (SigmaStat, SPSS Inc.). Molecular data were analyzed using 1-way ANOVA. A significant difference between groups was defined as $P$ less than 0.05 .

Study approval. The surgical facility met the American Association for the Accreditation of Laboratory Animal Care standards and the protocol was approved by the Vanderbilt IACUC.

\section{Author contributions}

DSE and ADC designed the studies, DSE, GK, and BF conducted the experiments, BF and PEW performed the surgeries, DSE, MS, KCC, RLP, and RMO acquired and analyzed data, and DSE and ADC wrote the manuscript.

\section{Acknowledgments}

This work was funded by National Institute of Diabetes and Digestive and Kidney Diseases grant R01-DK-18243. Hormone analysis was performed by Vanderbilt's Hormone Assay and Analytical Services Core (supported by the Vanderbilt DRTC and MMPC grants DK-20593 and DK-59637, respectively), and surgical and experimental expertise were provided by Vanderbilt's Large Animal Core (supported by DK-20593).

Address correspondence to: Dale S. Edgerton, Molecular Physiology and Biophysics, Vanderbilt University School of Medicine, Nashville, Tennessee 37232-0615, USA. Phone: 615.343.3193; E-mail: dale.edgerton@ vanderbilt.edu.

1. Edgerton DS, et al. Insulin's direct effects on the liver dominate the control of hepatic glucose production. J Clin Invest. 2006;116(2):521-527.

2. Hausler N, et al. Effects of insulin and cytosolic redox state on glucose production pathways in the isolated perfused mouse liver measured by integrated ${ }^{2} \mathrm{H}$ and ${ }^{13} \mathrm{C}$ NMR. Biochem J. 2006;394(Pt 2):465-473.

3. Maheux P, Chen YD, Polonsky KS, Reaven GM. Evidence that insulin can directly inhibit hepatic glucose production. Diabetologia. 1997;40(11):1300-1306.

4. Pilkis SJ, el-Maghrabi MR, Claus TH. Hormonal regulation of hepatic gluconeogenesis and glycolysis. Annu Rev Biochem. 1988;57:755-783.

5. Sindelar DK, Balcom JH, Chu CA, Neal DW, Cherrington AD. A comparison of the effects of selective increases in peripheral or portal insulin on hepatic glucose production in the conscious dog. Diabetes. 1996;45(11):1594-1604.

6. Sindelar DK, Chu CA, Venson P, Donahue EP, Neal DW, Cherrington AD. Basal hepatic glucose production is regulated by the portal vein insulin concentration. Diabetes. 1998;47(4):523-529.

7. Boden G, Cheung P, Homko C. Effects of acute insulin excess and deficiency on gluconeogenesis and glycogenolysis in type 1 diabetes. Diabetes. 2003;52(1):133-137.

8. Edgerton DS, et al. Small increases in insulin inhibit hepatic glucose production solely caused by an effect on glycogen metabolism. Diabetes. 2001;50(8):1872-1882.

9. Ramnanan CJ, et al. Molecular characterization of insulin-mediated suppression of hepatic glucose production in vivo. Diabetes. 2010;59(6):1302-1311.

10. Lewis GF, Vranic M, Harley P, Giacca A. Fatty acids mediate the acute extrahepatic effects of insulin on hepatic glucose pro- 
duction in humans. Diabetes. 1997;46(7):1111-1119.

11. Perry RJ, et al. Hepatic acetyl CoA links adipose tissue inflammation to hepatic insulin resistance and type 2 diabetes. Cell. 2015;160(4):745-758.

12. Rebrin K, Steil GM, Mittelman SD, Bergman RN. Causal linkage between insulin suppression of lipolysis and suppression of liver glucose output in dogs. J Clin Invest. 1996;98(3):741-749.

13. Sindelar DK, Chu CA, Rohlie M, Neal DW, Swift LL, Cherrington AD. The role of fatty acids in mediating the effects of peripheral insulin on hepatic glucose production in the conscious dog. Diabetes. 1997;46(2):187-196.

14. Titchenell PM, et al. Direct hepatocyte insulin signaling is required for lipogenesis but is dispensable for the suppression of glucose production. Cell Metab. 2016;23(6):1154-1166.

15. Cherrington AD. Banting Lecture 1997. Control of glucose uptake and release by the liver in vivo. Diabetes. 1999;48(5):1198-1214.

16. Lewis GF, Vranic M, Giacca A. Glucagon enhances the direct suppressive effect of insulin on hepatic glucose production in humans. Am J Physiol. 1997;272(3 Pt 1):E371-E378.

17. Obici S, Zhang BB, Karkanias G, Rossetti L. Hypothalamic insulin signaling is required for inhibition of glucose production. Nat Med. 2002;8(12):1376-1382.

18. Pocai A, et al. Hypothalamic K(ATP) channels control hepatic glucose production. Nature. 2005;434(7036):1026-1031.

19. Lewis GF, Zinman B, Groenewoud Y, Vranic M, Giacca A. Hepatic glucose production is regulated both by direct hepatic and extrahepatic effects of insulin in humans. Diabetes. 1996;45(4):454-462.

20. Fisher SJ, Kahn CR. Insulin signaling is required for insulin's direct and indirect action on hepatic glucose production. J Clin Invest. 2003;111(4):463-468.

21. Cherrington AD, Liljenquist JE, Shulman GI, Williams PE, Lacy WW. Importance of hypoglycemia-induced glucose production during isolated glucagon deficiency. Am J Physiol. 1979;236(3):E263-E271.

22. Ramnanan CJ, et al. Brain insulin action augments hepatic glycogen synthesis without suppressing glucose production or gluconeogenesis in dogs. J Clin Invest. 2011;121(9):3713-3723.

23. Greco AV, et al. Insulin and glucagon concentrations in portal and peripheral veins in patients with hepatic cirrhosis. Diabetologia. 1979;17(1):23-28.

24. Horwitz DL, Starr JI, Mako ME, Blackard WG, Rubenstein AH. Proinsulin, insulin, and C-peptide concentrations in human portal and peripheral blood. J Clin Invest. 1975;55(6):1278-1283.

25. Edgerton DS, et al. Changes in glucose and fat metabolism in response to the administration of a hepato-preferential insulin analog. Diabetes. 2014;63(11):3946-3954.

26. Farmer TD, et al. Comparison of the physiological relevance of systemic vs. portal insulin delivery to evaluate whole body glucose flux during an insulin clamp. Am J Physiol Endocrinol Metab. 2015;308(3):E206-E222.

27. Prager R, Wallace P, Olefsky JM. Direct and indirect effects of insulin to inhibit hepatic glucose output in obese subjects. Diabetes. 1987;36(5):607-611.

28. Buettner C, et al. Severe impairment in liver insulin signaling fails to alter hepatic insulin action in conscious mice. J Clin Invest. 2005;115(5):1306-1313.

29. Okamoto H, Obici S, Accili D, Rossetti L. Restoration of liver insulin signaling in Insr knockout mice fails to normalize hepatic insulin action. J Clin Invest. 2005;115(5):1314-1322.

30. Chandramouli V, Ekberg K, Schumann WC, Kalhan SC, Wahren J, Landau BR. Quantifying gluconeogenesis during fasting. Am J Physiol. 1997;273(6 Pt 1):E1209-E1215.

31. Edgerton DS, et al. Effects of insulin on the metabolic control of hepatic gluconeogenesis in vivo. Diabetes. 2009;58(12):2766-2775.

32. Rothman DL, Magnusson I, Katz LD, Shulman RG, Shulman GI. Quantitation of hepatic glycogenolysis and gluconeogenesis in fasting humans with ${ }^{13} \mathrm{C}$ NMR. Science. 1991;254(5031):573-576.

33. Chen C, Williams PF, Cooney GJ, Caterson ID, Turtle JR. The effects of fasting and refeeding on liver glycogen synthase and phosphorylase in obese and lean mice. Horm Metab Res. 1992;24(4):161-166.

34. Braun M, Ramracheya R, Rorsman P. Autocrine regulation of insulin secretion. Diabetes Obes Metab. 2012;14 Supp1 3:143-151.

35. Leibiger IB, Leibiger B, Berggren PO. Insulin signaling in the pancreatic beta-cell. Annu Rev Nutr. 2008;28:233-251.

36. Rhodes CJ, White MF, Leahy JL, Kahn SE. Direct autocrine action of insulin on $\beta$-cells: does it make physiological sense? Diabetes. 2013;62(7):2157-2163.

37. Duncan RE, Ahmadian M, Jaworski K, Sarkadi-Nagy E, Sul HS. Regulation of lipolysis in adipocytes. Annu Rev Nutr. 2007;27:79-101.

38. Gromada J, Franklin I, Wollheim CB. Alpha-cells of the endocrine pancreas: 35 years of research but the enigma remains. Endocr Rev. 2007;28(1):84-116.

39. Quesada I, Tudurí E, Ripoll C, Nadal A. Physiology of the pancreatic alpha-cell and glucagon secretion: role in glucose homeostasis and diabetes. J Endocrinol. 2008;199(1):5-19.

40. Inoue H, et al. Role of hepatic STAT3 in brain-insulin action on hepatic glucose production. Cell Metab. 2006;3(4):267-275.

41. Koch L, et al. Central insulin action regulates peripheral glucose and fat metabolism in mice. J Clin Invest. 2008;118(6):2132-2147.

42. Ramnanan CJ, et al. Interaction between the central and peripheral effects of insulin in controlling hepatic glucose metabolism in the conscious dog. Diabetes. 2013;62(1):74-84.

43. Girard J. The inhibitory effects of insulin on hepatic glucose production are both direct and indirect. Diabetes. 2006;55:S65-SS9

44. Grayson BE, Seeley RJ, Sandoval DA. Wired on sugar: the role of the CNS in the regulation of glucose homeostasis. Nat Rev Neurosci. 2013;14(1):24-37.

45. Könner AC, et al. Insulin action in AgRP-expressing neurons is required for suppression of hepatic glucose production. Cell Metab. 2007;5(6):438-449.

46. Lin HV, et al. Divergent regulation of energy expenditure and hepatic glucose production by insulin receptor in agouti-related protein and POMC neurons. Diabetes. 2010;59(2):337-346.

47. Vogt MC, Brüning JC. CNS insulin signaling in the control of energy homeostasis and glucose metabolism - from embryo to old age. Trends Endocrinol Metab. 2013;24(2):76-84. 
48. Coomans CP, et al. Stimulatory effect of insulin on glucose uptake by muscle involves the central nervous system in insulinsensitive mice. Diabetes. 2011;60(12):3132-3140.

49. Coomans CP, et al. Circulating insulin stimulates fatty acid retention in white adipose tissue via KATP channel activation in the central nervous system only in insulin-sensitive mice. J Lipid Res. 2011;52(9):1712-1722.

50. Mittelman SD, Fu YY, Rebrin K, Steil G, Bergman RN. Indirect effect of insulin to suppress endogenous glucose production is dominant, even with hyperglucagonemia. J Clin Invest. 1997;100(12):3121-3130.

51. Jurczak MJ, et al. Dissociation of inositol-requiring enzyme (IRE1 $\alpha$ )-mediated c-Jun N-terminal kinase activation from hepatic insulin resistance in conditional X-box-binding protein-1 (XBP1) knock-out mice. J Biol Chem. 2012;287(4):2558-2567.

52. Filippi BM, Yang CS, Tang C, Lam TK. Insulin activates Erk1/2 signaling in the dorsal vagal complex to inhibit glucose production. Cell Metab. 2012;16(4):500-510.

53. Persaud SJ, Asare-Anane H, Jones PM. Insulin receptor activation inhibits insulin secretion from human islets of Langerhans FEBS Lett. 2002;510(3):225-228.

54. Kawamori D, et al. Insulin signaling in alpha cells modulates glucagon secretion in vivo. Cell Metab. 2009;9(4):350-361.

55. Paranjape SA, et al. Influence of insulin in the ventromedial hypothalamus on pancreatic glucagon secretion in vivo. Diabetes. 2010;59(6):1521-1527.

56. Scherer T, et al. Brain insulin controls adipose tissue lipolysis and lipogenesis. Cell Metab. 2011;13(2):183-194.

57. Chen M, Woods SC, Porte D. Effect of cerebral intraventricular insulin on pancreatic insulin secretion in the dog. Diabetes. 1975;24(10):910-914

58. Yang TT, Ling SM, Tsao CW, Cheng JT. Opening of ATP-sensitive potassium channel by insulin in the brain-induced insulin secretion in Wistar rats. Horm Metab Res. 2010;42(2):110-114.

59. Dash S, Xiao C, Morgantini C, Koulajian K, Lewis GF. Intranasal insulin suppresses endogenous glucose production in humans compared with placebo in the presence of similar venous insulin concentrations. Diabetes. 2015;64(3):766-774.

60. Bollheimer LC, et al. Stimulatory short-term effects of free fatty acids on glucagon secretion at low to normal glucose concentrations. Metab Clin Exp. 2004;53(11):1443-1448.

61. Stagner J, Samols E, Polonsky K, Pugh W. Lack of direct inhibition of insulin secretion by exogenous insulin in the canine pancreas. J Clin Invest. 1986;78(5):1193-1198.

62. Boden G. Free fatty acids and insulin secretion in humans. Curr Diab Rep. 2005;5(3):167-170.

63. Moore MC, Coate KC, Winnick JJ, An Z, Cherrington AD. Regulation of hepatic glucose uptake and storage in vivo. Adv Nutr. 2012;3(3):286-294.

64. Moore MC, Connolly CC, Cherrington AD. Autoregulation of hepatic glucose production. Eur J Endocrinol. 1998;138(3):240-248. 